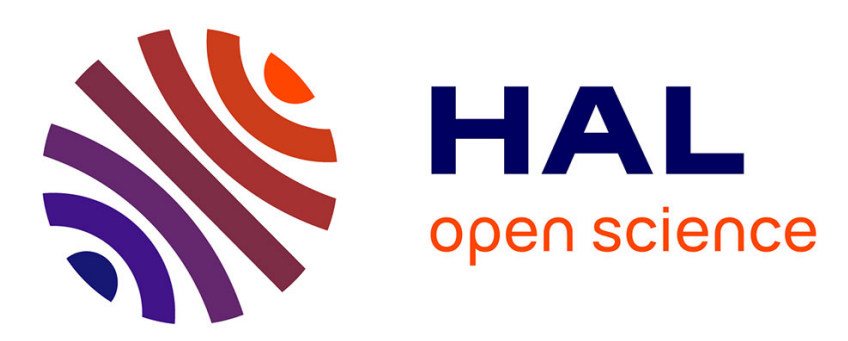

\title{
Concurrent Aerodynamic Optimization of Rotor Blades Using a Nash Game Method
}

\author{
Enric Roca Leon, Arnaud Le Pape, Jean-Antoine Désidéri, David Alfano, \\ Michel Costes
}

\section{To cite this version:}

Enric Roca Leon, Arnaud Le Pape, Jean-Antoine Désidéri, David Alfano, Michel Costes. Concurrent Aerodynamic Optimization of Rotor Blades Using a Nash Game Method. AHS 69th Annual Forum, American Helicopter Society, May 2013, Phoenix, Arizona, United States. hal-00934710

\author{
HAL Id: hal-00934710 \\ https://hal.inria.fr/hal-00934710
}

Submitted on 22 Jan 2014

HAL is a multi-disciplinary open access archive for the deposit and dissemination of scientific research documents, whether they are published or not. The documents may come from teaching and research institutions in France or abroad, or from public or private research centers.
L'archive ouverte pluridisciplinaire HAL, est destinée au dépôt et à la diffusion de documents scientifiques de niveau recherche, publiés ou non, émanant des établissements d'enseignement et de recherche français ou étrangers, des laboratoires publics ou privés. 


\title{
Concurrent Aerodynamic Optimization of Rotor Blades Using a Nash Game Method
}

\author{
Enric Roca León \\ Ph.D. Candidate, \\ Airbus Helicopter / ONERA, \\ Meudon, France \\ Arnaud Le Pape \\ Research Engineer, \\ ONERA, \\ Meudon, France \\ Jean-Antoine Désidéri \\ Research Director, \\ INRIA, \\ Sophia Antipolis, France \\ David Alfano \\ External Aerodynamics Leader, \\ Airbus Helicopter, \\ Marignane, France \\ Michel Costes \\ Research Engineer, \\ ONERA, \\ Meudon, France
}

Presented at the AHS 69th Annual Forum, Phoenix, Arizona, May 21-23, 2013. 


\begin{abstract}
A multi-objective strategy adapted to the aerodynamic concurrent optimization of helicopter rotor blades is developed. The present strategy is based on Nash Games from game theory, where the objective functions are minimized by virtual players involved in a non-cooperative concurrent game. A method is presented to split the design vector into two sub-spaces, defined to be the strategies of the players in charge of the minimization of the primary and the secondary objective functions respectively. This split of territory allows the optimization of the secondary function while causing the least possible degradation of the first one. This methodology is applied to the model rotor ERATO, seeking to maximize the Figure of Merit in hover while minimizing the required rotor power in forward flight. An initial constrained optimization in hover is conducted using a previously developed adjoint-based technique using the 3D Navier-Stokes solver elsA along with the gradient-based CONMIN algorithm. The chord, twist and sweep distributions of the baseline blade are parametrized using Bézier and cubic splines for a total of 16 design variables. The obtained optimized rotor is then used as a starting point to launch constrained and unconstrained Nash games. The comprehensive rotor code HOST is used to evaluate forward flight performance and a surrogate model is built to obtain the hover performance at low computational cost. Twist and sweep distribution laws are optimized independently at first and then a final joint optimization involving twist, sweep and chord is performed. The results demonstrate the potential of this technique to obtain helicopter rotor designs realizing interesting trade-offs between strongly antagonistic objectives.
\end{abstract}

\title{
Nomenclature
}

$\begin{array}{ll}c & \text { Mean chord }[\mathrm{m}] \\ C_{b}=\frac{100 C}{\frac{1}{2} \rho_{\infty} S \sigma R(R \Omega)^{2}} & \text { Rotor torque coefficient } \\ F M=\frac{Z_{b}^{3 / 2}}{20 C_{b}} \sqrt{\sigma} & \text { Figure of Merit } \\ N_{b} & \text { Number of blades } \\ P & \text { Required rotor power }[\mathrm{kW}] \\ R & \text { Rotor radius }[\mathrm{m}]\end{array}$




$\begin{array}{ll}S=\pi R^{2} & \text { Rotor disk surface }\left[\mathrm{m}^{2}\right] \\ Z_{b}=\frac{100 F z}{\frac{1}{2} \rho_{\infty} S \sigma(R \Omega)^{2}} & \text { Rotor thrust coefficient } \\ \mu=V_{\infty} /(\Omega R) & \text { Advance ratio } \\ \Omega & \text { Rotor rotational speed }[\mathrm{rad} / \mathrm{s}] \\ \sigma=\frac{N_{b} c}{\pi R} & \text { Rotor solidity }\end{array}$

\section{Introduction}

The aerodynamic optimization of helicopter rotor blades is a complex multidisciplinary problem that has been the subject of considerable research in the last decades. Historically, gradient descent methods for single objective optimizations were among the first ones to be used, such as the work presented by Welsh (Ref. 1) at NASA and (Ref. Zibi 2) at ONERA. These methods are still widely employed due to the limited number of simulations they usually need to reach a local minimum of the objective function (Refs. 1,3). The use of these algorithms was traditionally constrained to optimizations in hover employing low-fidelity models and/or a small number of design variables due to the prohibitive cost of computing the objective function gradient via finite differences with high-fidelity methods. The formulation of the discrete steady adjoint of the RANS equations has allowed the efficient use of gradient based algorithms and high-fidelity models in hover optimizations (Ref. 4), as the cost of the gradient evaluation becomes practically independent of the number of design parameters. A more complex problem is posed for optimizations in forward flight. First, the use of the adjoint formulation in unsteady flows becomes complicated, as it requires either considering the problem as periodic in order to use a steady adjoint formulation as shown by Choi (Ref. 5), or solving the unsteady adjoint equation backwards in time. In addition to rotor trim, rotor blades undergo important deformations in forward flight and thus fluid-structure interaction needs to be taken into account, especially for swept blades. This implies the use of structural models to include the deformations and usually requires coupling with the aerodynamic solver to obtain a converged solution, further increasing the computational cost associated to the evaluations, as presented by Imiela (Ref. 6).

An alternative to gradient based methods is the use of stochastic or genetic algorithms, even if this comes 
at an extremely high computational cost (specially in aerodynamics). However, parallel computing and the use of surrogate based optimization techniques can alleviate this problem, as shown in the works of Barakos (Ref. 7), Glaz (Refs. 8,9) and more recently Leusink (Ref. 10).

Furthermore, designers need to take into consideration multiple relevant criteria when optimizing the shape of rotor blades. Typically it is required to find a compromise between optimal hover and forward flight configurations, which usually are antagonistic (i.e. improving hover performance implies worsening forward flight characteristics). This leads to multi-objective optimization problems. The most common approach to this problem is the use of an agglomerated function (Refs. 6,11), where all the criteria are grouped in a single functional using weighting coefficients for each of the objectives. The advantage of this method is its low computational cost. However, the obvious issue is that the practitioner must set the weights which usually leads to some arbitrariness in their choice. The opposite approach consists in identifying the Pareto equilibrium front, which is costly to compute but provides the practitioner with very complete information to support the decision making, even if there is a lack of hierarchy between the compromise solutions. Another constraint of these methods is that usually only a small degradation of the performance of an optimum or baseline configuration is acceptable when introducing other criteria.

This paper presents an innovative alternative treatment of multi-objective concurrent problems. This method does not need the adjustment of weighting constants nor it is as computationally expensive as finding the Pareto front. The problem is formulated as a classical Nash Game (Ref. 12), which is a noncooperative game between multiple players (each one of them representing an objective function) where each participant simultaneously tries to improve their objective independently of the other players, using a portion of the design territory. This method can be applied at the end of a successful single objective optimization in order to optimize a secondary criterion without degrading in excess the gains already achieved in terms of primary criterion.

This methodology is adapted for those cases in which two antagonistic objectives are considered (such as the aerodynamic requirements of hover and forward flight). In this paper the described methodology to optimize an helicopter rotor in hover and forward flight is presented and demonstrated on the ERATO blade (Ref. 13), which presents a complex planform geometry including forward and backward sweep. An initial hover optimization using the discrete adjoint technique is performed employing elsA (Ref. 14), 
a high-fidelity Computational Fluid Dynamics (CFD) code developed at ONERA. Subsequently, multiple Nash Games are presented optimizing twist, chord and sweep laws in forward flight. The HOST code (Ref. 15) is used to evaluate the performance in forward flight and a surrogate model is used as a substitute of the expensive CFD evaluations during the Nash Game. The optimization results are discussed and analyzed.

\section{Optimization Strategy}

Both single objective optimizations of the hover configuration and multi-objective optimizations involving hover and forward flight are presented in this paper. In the following, the chosen strategies for each type of problem are detailed.

\section{Single-objective: Adjoint-based Optimization}

Single objective optimizations in hover are conducted using a gradient-based method coupled with a discrete adjoint of the Reynolds-averaged Navier-Stokes (RANS) equations, reproducing the procedure presented by Dumont (Ref. 4). The chosen descent algorithm is CONMIN, a classic gradient-based algorithm developed by Vanderplaats (Ref. 16) and included in DAKOTA, the open-source optimizing tool developed by Sandia National Laboratories (Ref. 17). The choice of a gradient based optimizer was motivated by the fact that the adjoint solver allowed the computation of gradients at a minimal cost. Indeed, the gradient of the functional with respect to all the parameters can be obtained at the cost of roughly one function evaluation.

\section{Multi-objective: Nash game with territory splitting}

The multi-objective problem can be modeled as a classic Nash Game, where the hover and forward flight performance are represented by 2 virtual players or disciplines, namely $J_{A}$ and $J_{B}$, that play a non-cooperative concurrent game. Both disciplines share the design vector $Y=\left(Y_{A}, Y_{B}\right)$. During the game, each subvector $Y_{i}$ is assigned to its respective player $J_{i}$, who tries to improve its objective using 
its portion of the design territory while taking into account the actions of their opponent (i.e. the other discipline's subvector), as proposed by Tang, Désidéri and Périaux (Ref. 18). A Nash equilibrium $\hat{Y}$ is reached when no further improvements are obtained for each player if their opponent keeps its strategy unchanged, verifying:

$$
\begin{array}{r}
\hat{Y}=\left(\hat{Y}_{A}, \hat{Y}_{B}\right) \\
\hat{Y}_{A}=\operatorname{Argmin}_{Y_{A}}\left(Y_{A}, \hat{Y}_{B}\right) \\
\hat{Y}_{B}=\operatorname{Argmin}_{Y_{B}}\left(\hat{Y}_{A}, Y_{B}\right)
\end{array}
$$

This equilibrium can be obtained by an iterative process in which each discipline runs a small number of optimizing iterations before exchanging information with the other discipline, until convergence (or a relaxed convergence) is obtained. In pseudo-code this process can be described as:

1) Initialize the design vector: $Y=\left(Y_{A}^{i}, Y_{B}^{i}\right)$, with $i=0$.

2) Run independent parallel optimizations on $J_{A}$ and $J_{B}$ :

Player $A$ : $Y=\left(Y_{A}, Y_{B}^{i}\right)$. Perform $K_{A}$ iterations for the optimization problem $\min J_{A}\left(Y_{A}, Y_{B}^{i}\right)$ to obtain $Y_{A}^{i+1}$.

Player B: $Y=\left(Y_{A}^{i}, Y_{B}\right)$. Perform $K_{B}$ iterations for the optimization problem $\min J_{B}\left(Y_{A}^{i}, Y_{B}\right)$ to obtain $Y_{B}^{i+1}$.

3) Update the design vector: $Y=\left(Y_{A}^{i+1}, Y_{B}^{i+1}\right)$

4) Test for convergence:

if $\left(Y_{A}^{i+1}, Y_{B}^{i}\right) \approx\left(Y_{A}^{i}, Y_{B}^{i+1}\right):$ stop.

else: go back to Step 2 using $Y_{A}^{i}=Y_{A}^{i+1}$ and $Y_{B}^{i}=Y_{B}^{i+1}$.

Relaxation of the convergence criterion is used in order to guarantee the convergence of the algorithm. In practice, convergence is assumed when either the squared difference of the L-2 norm of the scaled design vectors is smaller than a certain positive $\delta$ (Eq. 2) or when a maximum number of exchanges between disciplines is reached (Eq. 3). Scaling of the design vectors for the test is necessary in order to account 
for the difference of magnitudes between the design variables.

$$
\begin{array}{r}
\left(\left\|\left(Y_{A}^{i+1}, Y_{B}^{i}\right)\right\|-\left\|\left(Y_{A}^{i}, Y_{B}^{i+1}\right)\right\|\right)^{2} \leq \delta \\
i \geq N_{\max }
\end{array}
$$

This method requires the partition of the design variable space, which determines the equilibrium solution. This split of the design vector arguably introduces some arbitrariness. However, an innovating theory to split the variables territory has been recently proposed (Ref. 19) for the treatment of concurrent optimization cases, which is hereby applied.

In this framework we consider the case where two disciplines must be optimized with one being regarded as principal. While optimizing the secondary criterion, sub-optimality for the principal discipline should be assured. In other words, after a successful single objective optimization of a principal discipline, a multi-objective competitive optimization is conducted, assuring that the gains obtained on the first optimization are not degraded in excess. The intended approach consists in conducting the optimization of the secondary discipline (i.e. $J_{B}$ ) as a small perturbation from the original optimum $Y_{J_{A}}^{*}$. The solution corresponds to a Nash equilibrium between the two disciplines. Precisely, for such an scenario the design vector (of length $N$ ) can be defined as:

$$
\begin{array}{r}
Y=Y(U, V)=Y_{J_{A}}^{*}+S\left(\begin{array}{c}
U \\
V
\end{array}\right) \\
U=\left(\begin{array}{c}
u_{1} \\
\vdots \\
u_{N-p}
\end{array}\right) \quad V=\left(\begin{array}{c}
v_{p} \\
\vdots \\
v_{N}
\end{array}\right)
\end{array}
$$

In which $Y_{J_{A}}^{*}$ corresponds to the previously found optimum of the principal discipline and $S$ is an invertible $N \mathrm{x} N$ matrix henceforth referred to as splitting matrix. The $U$ and $V$ subvectors correspond to the design vectors of the principal and secondary functionals respectively. The splitting matrix is defined such as small perturbations of the parameters about $Y_{J_{A}}^{*}$ that affect the secondary criterion cause the least possible degradation to $J_{A}$. 
At the end of the first optimization access to $J_{A}^{*}$, its gradient $\nabla J_{A}^{*}$ and the Hessian matrix $H_{A}^{*}$ via direct evaluation or surrogate models is supposed. Assuming that $Y_{A}^{*}$ is a local or global unconstrained minimum of the functional $J_{A}$, then $\nabla J_{A}^{*}=\overrightarrow{0}$ and the Hessian matrix $H_{A}^{*}$ is real and symmetric (hence all its eigenvalues $h_{i}$ are real and positive). In this case, the Hessian can be diagonalized (Eq. 7) obtaining the matrix $\Omega_{H}$ formed by the eigenvectors $\vec{\omega}_{i}$ of the Hessian.

$$
\begin{aligned}
H_{A}^{*} & =\Omega_{H} \Lambda_{H} \Omega_{H}^{T} \\
\Lambda_{H} & =\operatorname{Diag}\left(h_{i}\right) \\
\Omega_{H} & =\left(\cdots \overrightarrow{\omega_{i}} \ldots\right)
\end{aligned}
$$

The territory of the secondary functional should be taken to be the span of $p$ eigenvectors of the Hessian matrix associated with the smaller eigenvalues $h_{i}$. The eigenvalues are thus ordered as a monotone decreasing sequence verifying Eq. 9 and the splitting matrix $S$ is obtained reordering the eigenvectors according to the sorted eigenvalues (Eq. 10).

$$
\begin{array}{r}
h_{1} \geq h_{2} \geq \ldots \geq h_{N} \\
S=\left(\begin{array}{llll}
\vec{\omega}_{1} & \vec{\omega}_{2} & \ldots & \vec{\omega}_{N}
\end{array}\right)
\end{array}
$$

For the case of constrained problems an additional step is required. Assuming $\mathrm{K}$ active constraints, at the end of the first optimization we have access to the linearly independent constraint gradient vectors $\left\{L_{k}\right\}=\left\{\nabla g_{k}^{*}\right\}$. The Gram-Schmidt orthogonalization process is then applied to the vectors $\left\{L_{k}\right\}$ to define an orthonormal basis $\left\{m^{k}\right\}$. A new matrix $H_{A}^{\prime}$ is finally obtained via Eqs. 11, 12 and the same procedure described previously can be applied using $H_{A}^{\prime}$ instead of the original Hessian matrix $H_{A}$.

$$
\begin{array}{r}
P=I-\vec{m}^{1} \vec{m}^{1^{T}}-\vec{m}^{2} \vec{m}^{2^{T}}-\ldots-\vec{m}^{K} \vec{m}^{K^{T}} \\
H_{A}^{\prime}=P H_{A}^{*} P
\end{array}
$$

However, in the constrained case the first $K$ eigenvalues $h_{i}$ are by definition equal to zero, and must be assigned to the primary discipline. Thus, in order to obtain the splitting matrix $S$ the eigenvalues must be ordered placing first the zero-valued ones followed by a monotone decreasing sequence (as already defined in Eq. 9). 
It should be noted that in the case of aerodynamic shape optimization, the values of the design vector $Y$ correspond to shape control variables that are associated with spatial zones of the blade. Therefore, the splitting matrix reflects a physical choice of the territories which are the most influential for the principal discipline. Typically, in the case of hover optimizations this zone corresponds to the blade tip. In practice this territory splitting ensures that the steepest descent direction of $J_{A}^{*}$ is mostly controlled by the subvector $U$, leaving the least sensitive path with respect to $J_{A}$ to the secondary discipline $J_{B}$.

The search of the Nash equilibrium could be applied directly to the problem as defined in Eq. 1, but a redefinition of the multi-objective problem is proposed by Désidéri (Ref. 19), reproduced in Eqs. 13 and 14 for the case of a constrained problem.

$$
\begin{gathered}
\left\{\begin{array}{c}
\min _{U \in \mathbb{R}^{N-p}} J_{A}[Y(U, \hat{V})] \\
\text { Subject to: } g=0
\end{array}\right. \\
\min _{V \in \mathbb{R}^{p}} J_{A B}[Y(\hat{U}, V)]=\frac{J_{A}}{J_{A}^{*}}+\epsilon\left(\theta \frac{J_{B}}{J_{B}^{*}}-\frac{J_{A}}{J_{A}^{*}}\right)
\end{gathered}
$$

Fixed subvectors are represented by $\hat{U}$ and $\hat{V}, \epsilon$ is a continuation parameter comprised between 0 and 1 that allows a gradual introduction of the antagonism between disciplines and $\theta$ represents a relaxation factor which in practice is set to 1 . Using this formulation, a smooth continuum of Nash equilibria is found starting from the optimum of the principal discipline $J_{A}^{*}(\epsilon=0)$ and finishing for the case of a pure minimization of $J_{B}(\epsilon=1)$.

\section{Optimization Chain}

The aerodynamic optimizations have been implemented by updating preexisting optimization tools developed at ONERA (Refs. 4,11). The optimization loop is linked to multiple flow solvers (namely elsA, HOST and a variety of surrogate models), as well as to the open-source platform DAKOTA and the Nash game algorithm. An in-house mesh deformation tool is used to pre-process the geometry when launching CFD computations, while for the rest of the flow solvers it is computed analytically. When required by the optimization algorithm, gradients are computed via the adjoint method for CFD evaluations. In this case the adjoint computation is launched following a successful standard computation (i.e. once the flow 


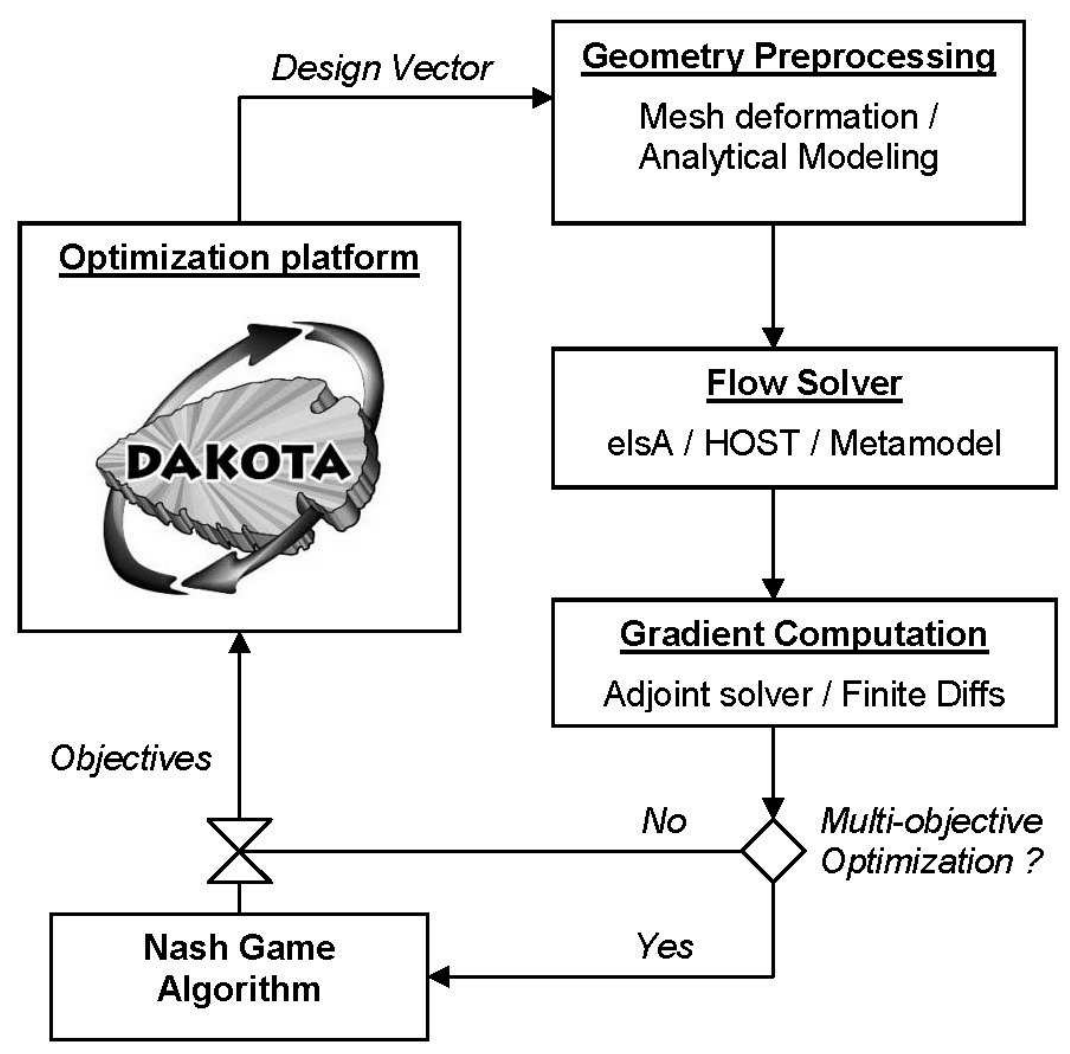

Fig. 1 Flow chart of the optimization process.

is converged). For the lower fidelity models the cost of the simulations is much more modest, allowing the gradient computation via forward or central finite differences. The flow-chart of the optimization loop is presented in Fig. 1.

When conducting multi-objective optimizations the Nash game method is employed and launches independent parallel optimizations for each of the concurring disciplines using the Dakota platform. Further details on the flow solvers are given in the following sections.

\section{Flow solvers}

\section{$\underline{\text { elsA }}$}

The elsA CFD code developed at ONERA solves the compressible 3-D RANS equations using a cell centered finite-volume method. The turbulence is modeled using the $k-\omega$ Kok model presented in (Ref. 20) with a shear stress transport (SST) correction (Ref. 21). The flux is discretized using a 2nd 
order Roe's scheme (Ref. 22) in order to assure consistency with the discrete adjoint solver, along with Van Albada limiter (Ref. 23). The time integration is performed using an implicit algorithm based on a backward Euler scheme. Due to the high computational cost associated with forward flight computations elsA is only used to evaluate the helicopter performance in hover assuming no deformations.

\section{$\underline{\text { HOST }}$}

Eurocopter's Helicopter Overall Simulation Tool (HOST) is a comprehensive code that considers the blade dynamics, using a 1D Euler-Bernoulli beam model, coupled with a simplified aerodynamics model based on lifting-line theory. The aerodynamic coefficients are determined via interpolation of 2D airfoil lookup tables, using the computed rotor trim information in order to evaluate the local Mach numbers and angles of attack. In the present work the induced velocities are modeled using the prescribed wake METAR model (Ref. 24). This code is used to quickly evaluate the performance of the rotor in forward flight, taking into account blade dynamics and deformations.

\section{Surrogate Model}

Given the considerable computational cost of evaluating the hover rotor performance with high fidelity codes an the need to compute the Hessian matrix, an alternative approach is used for the evaluation of the Figure of Merit in multi-objective optimizations. Indeed, a surrogate model is built to mimic the behavior of the physical high-fidelity model at a very low computational cost. In order to build a metamodel of the rotor hover performance a database of well chosen CFD evaluations is needed. In the present work, a Design of Experiments (DoE) technique known as Latin Hypercube Sampling (Ref. 25) is used to initialize the metamodel database. The surrogate model is built using a kriging technique with constant mean (Ref. 26) included in Dakota's library. Estimates of the metamodel error are computed using K-fold stratified cross-validation techniques, as recommended by Kohavi (Ref. 27) using the Kmeans stratification strategy proposed by Diamantidis (Ref. 28). This technique recursively uses groups of points of the training data in order to test the metamodel, which is built using the original database minus the test points, chosen so that they are not close in the design space (i.e. not clustered). An estimation of the bias and the variance of the metamodel can thus be obtained. An additional advantage of the kriging/gaussian process technique is that it provides an estimation of the model uncertainty. This characteristic is used to find the points that maximize the expected improvement of the functional and 
updating the metamodel with them, as described in the Efficient Global Optimization (EGO) method (Ref. 29). In this paper, the metamodel was iteratively updated with the maximum expected improvement point as well as the computed global minimum while monitoring the global bias, defined as the root mean square deviation. The iterative updating process of the metamodel was stopped either when acceptable values where reached (namely, mean errors of less than 1 point of the Figure of Merit) or when no further improvements were obtained for 3 consecutive iterations.

\section{Application on the ERATO blade}

The baseline rotor used throughout the computations was the ERATO rotor. This model rotor, developed in a joint program between Eurocopter, ONERA and DLR was designed to reduce noise emissions (Ref. 13). It features a $2.1 \mathrm{~m}$ radius, a mean chord of $0.14 \mathrm{~m}$ and a linear aerodynamic twist of $-10^{\circ} / R$. The blade planform has forward and backward sweep as well as a non-optimized straight tip.

The mesh strategy is the same for all hover simulations: a quarter of rotor mono-block mesh of 0.81 million points with a C-H grid topology (Fig. 2). As presented in (Ref. 4), this coarse grid is used to evaluate the FM, allowing better turnaround times for the evaluations in the present optimization exercise. Convergence is obtained after approximately 1500 iterations even though small oscillations (variations of the FM value of approximately 0.005 ) persist up to 2500 iterations. Typically, the hover computations require about 3000s of restitution time in parallel mode using 15 processors on a scalar calculator using Xeon 5500 cores.

\section{Variables and parametrization}

The blade is parametrized using Bézier and cubic Spline laws controlling twist and chord variations as well as the sweep distribution. Precisely, five control points are used to model a Bézier distribution of twist representing a delta with respect to the baseline rotor. The Bézier curve corresponding to chord controls the percentage variation of chord along the blade span. Sweep is modeled directly using a cubic spline, and finally the collective pitch is added as a variable in order to ensure that performance gains are obtained always with respect to the maximum of FM (independently of the rotor thrust value). The total 


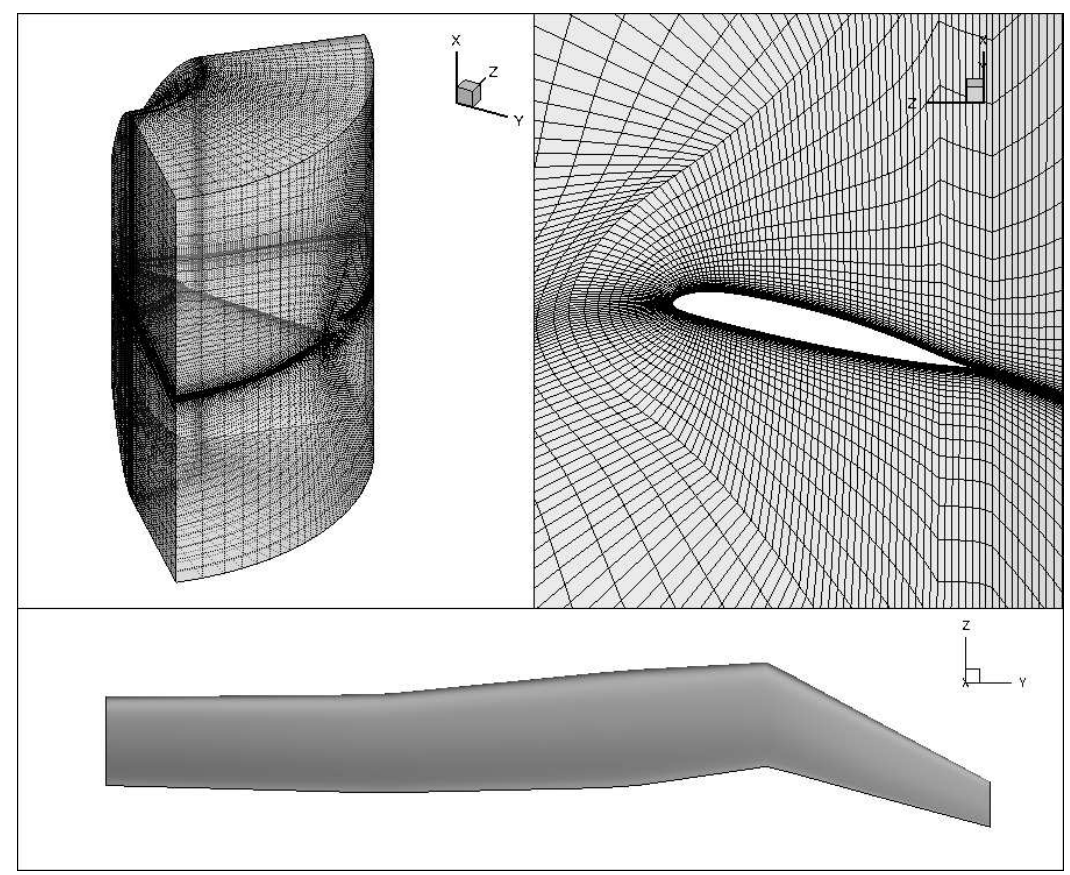

Fig. 2 Mesh strategy and ERATO blade geometry.

number of variables for hover optimization is therefore 16. In contrast, in forward flight optimization the collective pitch is a function of the rotor trim and is thus not taken into account. The position of the control points is shown in Fig. 3, the first control point being necessary to assure a smooth transition between the optimized zone and the original blade planform and not intervening as a variable in the optimization process.

\section{Objective Function and Constraints}

The objective of the hover optimization is to maximize the Figure of Merit, which represents the rotor efficiency in hover. The FM is a function of the thrust and torque coefficients, which in turn depend on the rotor solidity. In order to obtain comparable FM values between rotors, iso-solidity must be enforced. In particular, thrust-weighted solidity is forced to be constant throughout the optimization run (as recommended by Bingham (Ref. 30) and Dumont (Ref. 4)). The thrust weighted solidity is thus computed as:

$$
\sigma_{T W}=\frac{N_{b}\left(R_{\text {blade }}-R_{\text {hub }}\right)}{S} \frac{\int_{R_{\text {hub }}}^{R_{\text {blade }}} r^{2} c(r) d r}{\int_{R_{\text {hub }}}^{R_{\text {blade }}} r^{2} d r}
$$




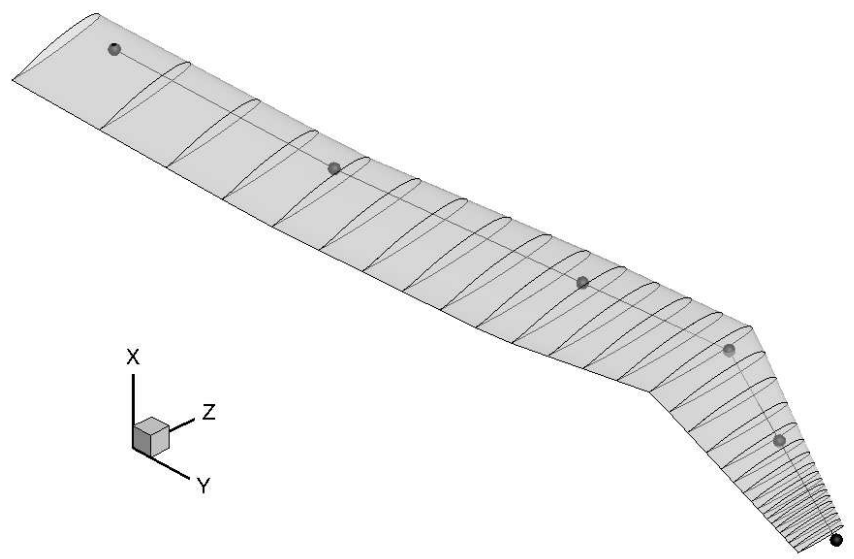

Fig. 3 Control point locations for the ERATO blade optimization.

This geometric requirement is directly integrated in the blade deformation module as an implicit constraint, avoiding solutions with very fine overloaded tips.

In forward flight, the required rotor power $(P)$ is the chosen function to be minimized. In forward flight computations the rotor is trimmed imposing zero flapping (i.e. $\beta_{l c}=\beta_{l s}=0$ ) at a given thrust coefficient $Z_{b}$ and at fixed $\mu$ in order to be able to fairly compare different shaped blades. Precisely, the chosen design point for the ERATO rotor is at $\mu=0.344, Z_{b}=12.5$ and $C_{x} S=0.1$ for a tip Mach number $M_{t i p}=0.617$. A constraint is imposed on the maximum admissible value of static link loads in order to avoid unrealistic solutions with excessive sweep. However, it should be noted that no constraints are imposed on the dynamic properties of the blade and that the structural properties of the structure are not updated.

\section{Single Objective Optimization in Hover}

An optimization of the FM involving twist, chord and sweep distributions was conducted using the adjoint method along with the CONMIN algorithm. The twist variation at the blade tip was bounded in order to attain a maximum geometric twist of -12.5 degrees. The results presented in Fig. 4 show fast convergence towards a local maximum. The total cost of the optimization was of thirty-one CFD evaluations plus eight adjoint evaluations requiring a total restitution time of $33 \mathrm{CPU}$ hours. The resulting 


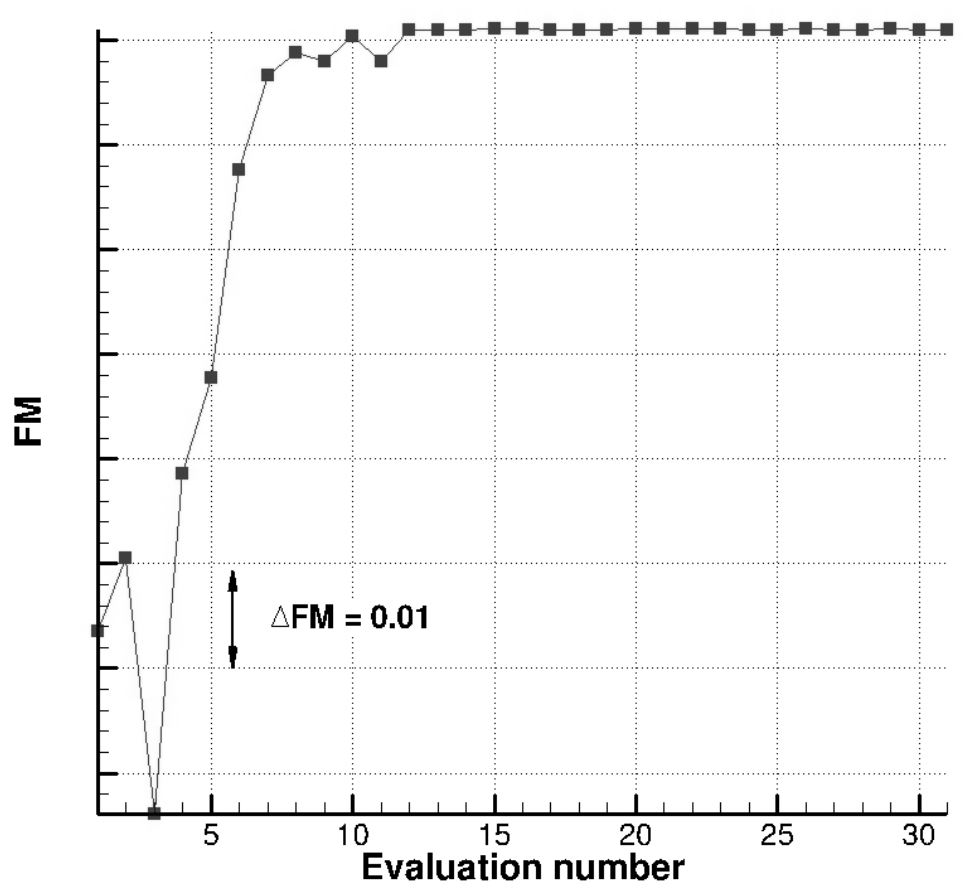

Fig. 4 Convergence history of the hover optimization.

twist, chord and sweep distributions are presented in Fig. 5.

The twist variation distribution remains positive for the inner part of the blade span and reaches the minimum fixed boundary at the tip. The chord is also reduced for the most part of the blade (as a result of the fixed thrust-weighted solidity) and it is strongly augmented at the rotor tip (+37\%). The sweep variation remains small, with a maximum deviation from the initial distribution of $-0.03 \mathrm{~m}$ at $0.8 \mathrm{R}$. The optimized rotor increases its maximum FM by 6.1 points, as it is observed in Fig. 6 as well as its load capacity.

The baseline rotor and the hover optimum are briefly compared in Figs. 7,8,9 at the point corresponding to the maximum of FM of the ERATO rotor and at iso-thrust coefficient for the optimized rotor. The lift and power coefficients are presented in 7. They show a reduction of the thrust at the rotor tip, which is regained in the inner part of the design. The thrust increase inboard allows for a more evenly lift distribution along the optimized blade. In addition, the consumed power is slightly increased between 0.75 and $0.8 \mathrm{R}$ but an important reduction is achieved in the blade tip zone. 


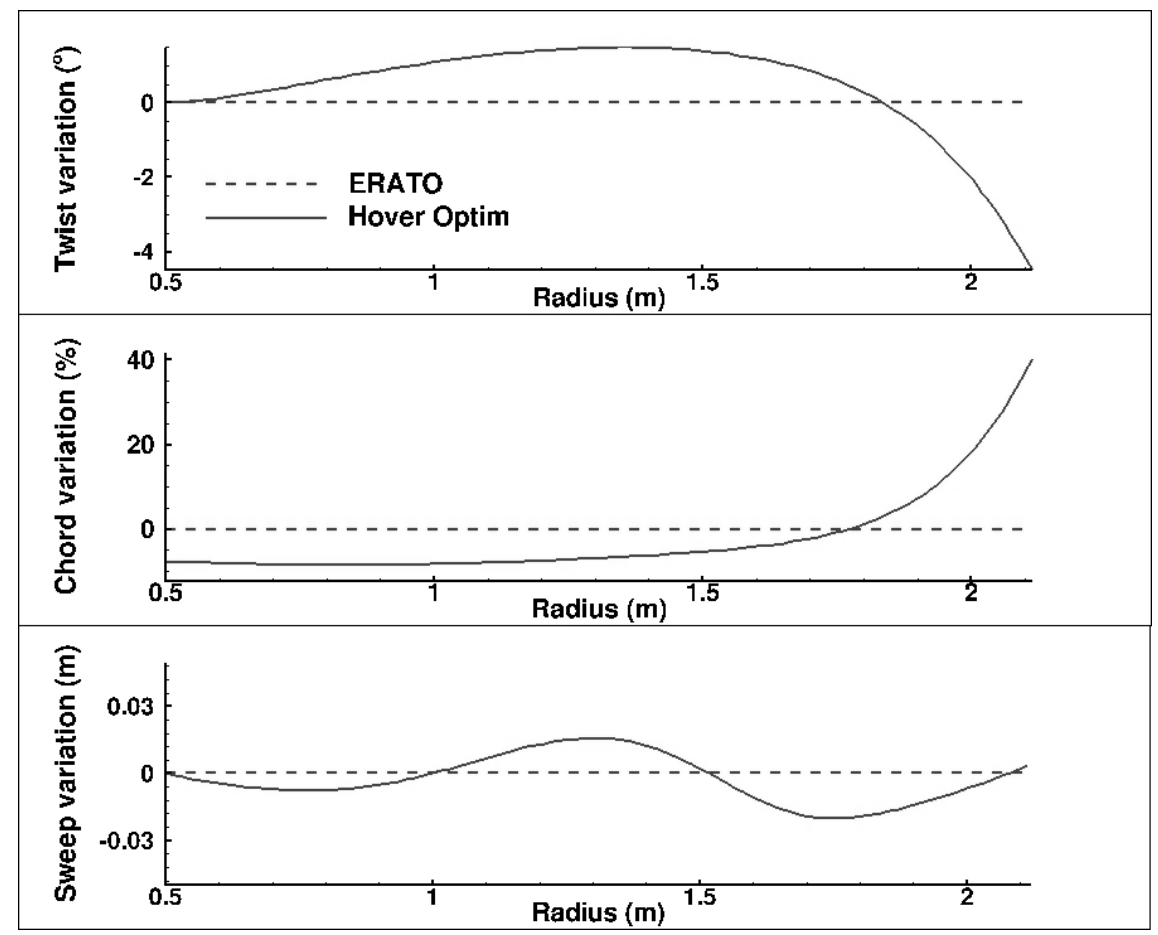

Fig. 5 Geometric twist, chord and sweep law variations of the optimum (continuous line) with respect to the baseline rotor (dashed line).

In Fig. 8 the pressure distribution is presented for 3 different blade sections. The image suggests that most of the gains are concentrated in the region of the blade tip, as expected. In particular, for sections near the tip where the flow is locally supersonic the optimization algorithm reduces the magnitude of $C_{P}$. The friction lines in Fig. 9 are shown for the baseline and optimized rotors at the maximum of FM and at the same thrust. The flow is locally separated at the baseline rotor tip while it remains attached for the optimized blade.

The chord and twist distributions follow the same trends as in previous similar works (Refs. 3,4).

\section{Multi-Objective Optimization: Hover and Forward Flight}

The obtained optimum in hover was used as a starting point to launch Nash Games with and without constraints seeking to improve the forward flight performance. 


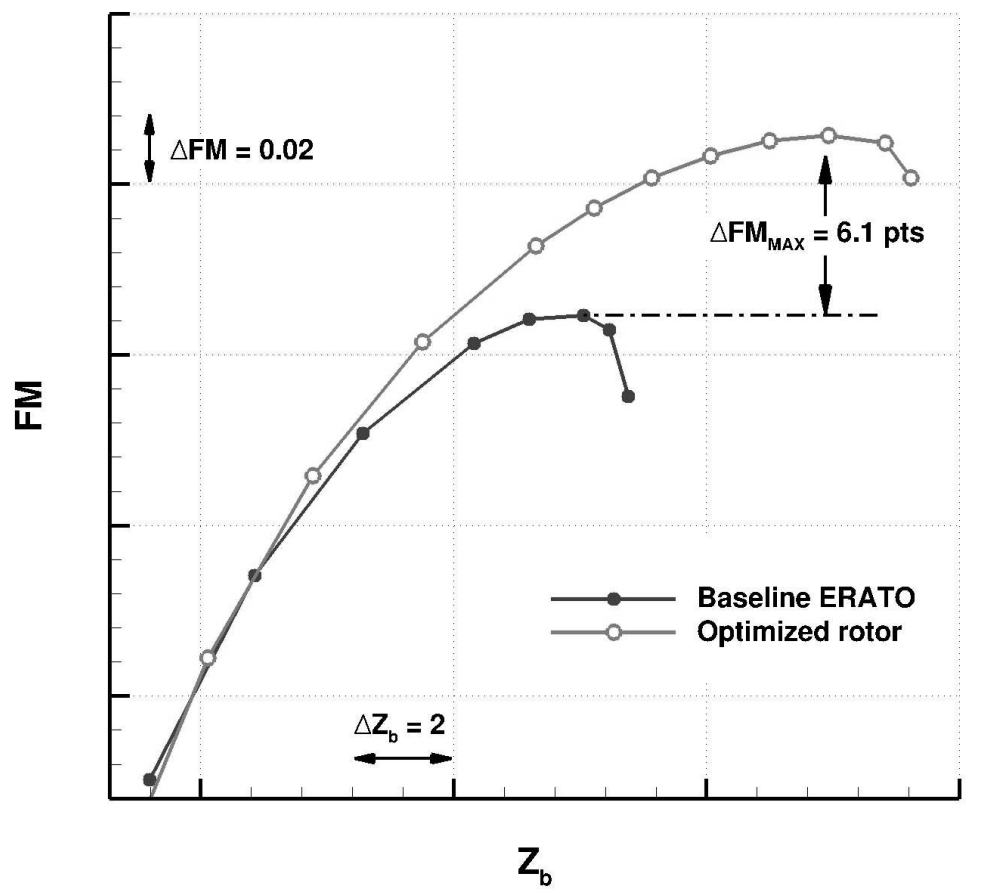

Fig. 6 Maximum FM gain for the optimum with respect to the baseline rotor. 

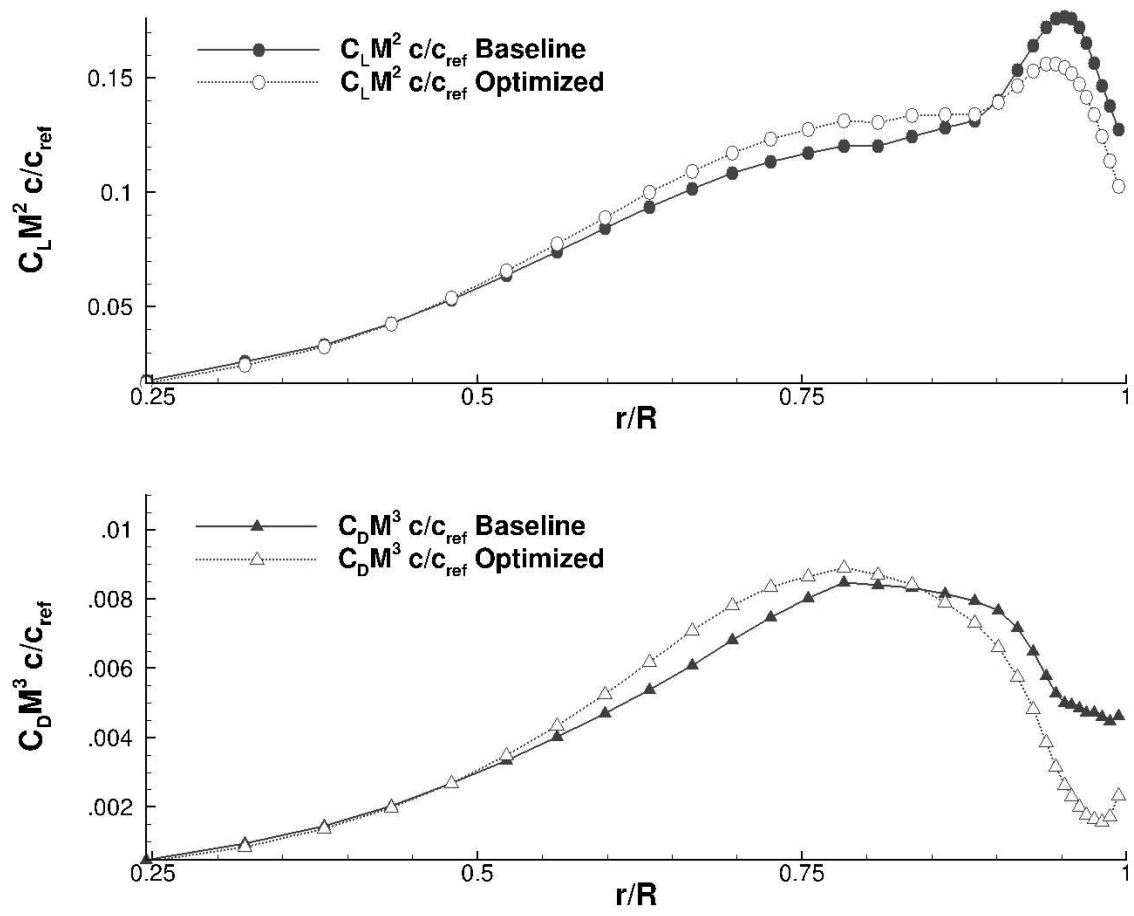

Fig. 7 Lift and power distribution along the blade span of the baseline and optimized rotor at the same thrust value.

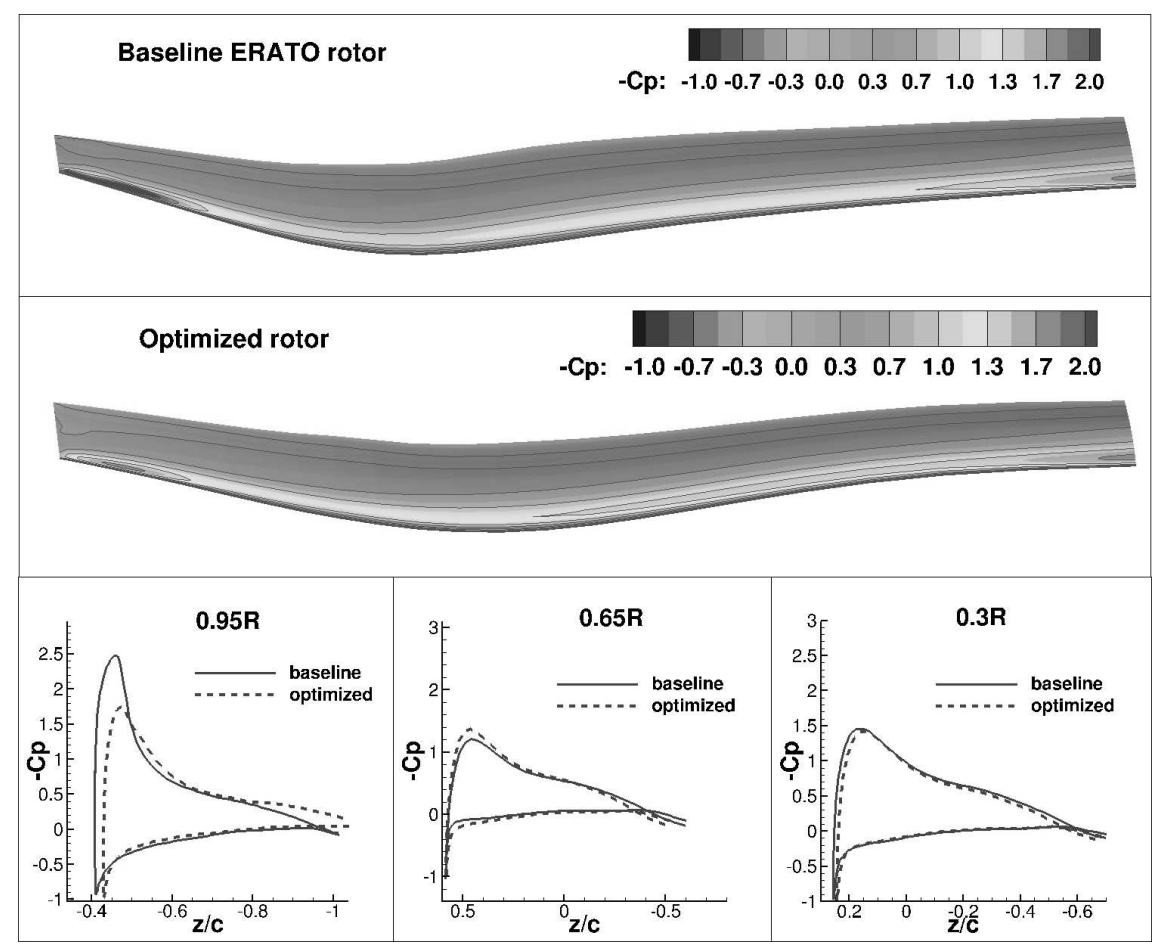

Fig. 8 Pressure coefficient contours for the baseline and optimized rotor at the same thrust value. 


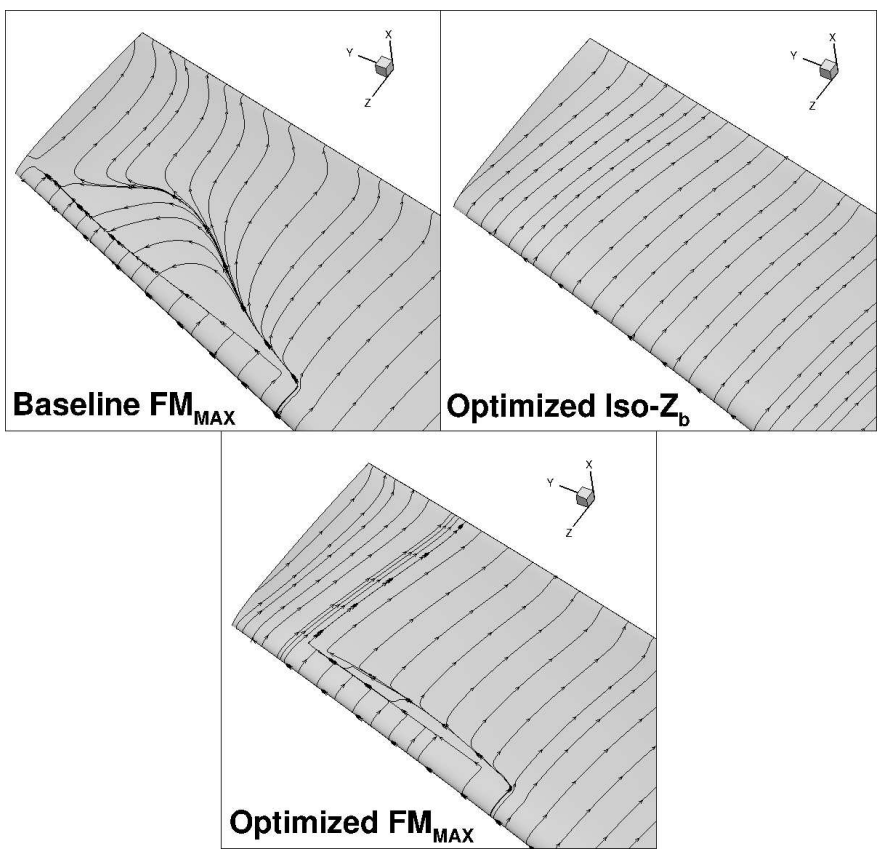

Fig. 9 Friction lines comparison at the blade tip for the thrust corresponding to the baseline $F M_{M A X}$ and at the optimized blade $F M_{M A X}$.

\section{Twist Optimization}

An unconstrained Nash Game involving the twist distribution was conducted using a kriging metamodel to evaluate the Hessian matrix at the optimum (necessary in order to split the design territories) and to obtain the hover performance. The geometric twist law was controlled using 5 Bézier poles. The surrogate model was built using an initial Latin Hypercube Sample (LHS) of 45 points chosen around the hover optimum plus the optimum itself, and was updated iteratively 5 times until a total of 56 points was reached. Out of the 5 variables, 2 were assigned to the primary discipline (hover performance, $J_{A}$ ) and 3 to the secondary (i.e. power consumption, $J_{B}$ ) using the previously described methodology. Results are shown in Fig. 10 for a complete run of the game, discretized in 10 Nash equilibria. At the equilibrium points each player conducted a couple of optimization iterations to optimize its function using their design vector subset before exchanging information with the opposite player once or twice depending on the convergence of the equilibrium. Thus, the forward flight solver was called to evaluate the functions 140 times and to evaluate the gradient 20 times. 
The curves shown in Fig. 10 are normalized with respect to the values at the initial hover optimum $J_{i} *$. The dashed lines $\left(J_{i 0} / J_{i} *\right)$ mark the baseline rotor performance, the upper line representing the initial Figure of Merit value (with respect to the hover optimum). Gains on hover performance are thus represented by values bellow this line. The lower dashed line represents the ERATO consumed power, with points signifying an increase in consumption being situated above the line. Finally, $\epsilon$ represents the continuation parameter introduced in Eq. 14.

The Nash equilibria obtained using the surrogate model were subsequently checked using CFD. A good agreement is found, especially near the initial hover optimum. The surrogate model performance is degraded as the Nash Game advances and the optimization algorithm searches farther in regions near the limit of validity of the metamodel. However, and most importantly, the metamodel trends are very similar to the ones shown by the high-fidelity code. The obtained continuum of Nash equilibria designs are close in the functional space, but also in the design space. Indeed, a single design vector is optimized instead of a population of vectors as is the case in genetic algorithms.

At the starting point the optimized blade in hover presents about a 25\% gain of the primary discipline (defined as $100(1-F M)$ ) with respect to ERATO, which translates to approximately a $8.7 \%$ of Figure of Merit gain and approximately a 5\% increase in the power consumption. The last point of the game continues to optimize the hover performance $(+3.45 \%$ increase) while attaining practically the same level of power consumption in forward flight as ERATO.

The variation of the twist law distributions of the hover optimum and the last Nash equilibrium of the game are shown in Fig. 11. The antagonistic nature of the objectives is reflected on this distribution: both points show opposite trends on most part of the blade. The blade tip twist distribution remains relatively unchanged. This is a direct consequence of the split of territories performed at the beginning of the Nash Game: indeed, the blade tip is very important for the hover performance, and thus the shape variations associated with this zone are mostly controlled by the principal discipline.

The performance variations of the hover optimum and the Nash compromise at $\epsilon=1$ with respect to ERATO are presented in Fig. 12. The total power repartition is globally reduced for the hover optimum at 


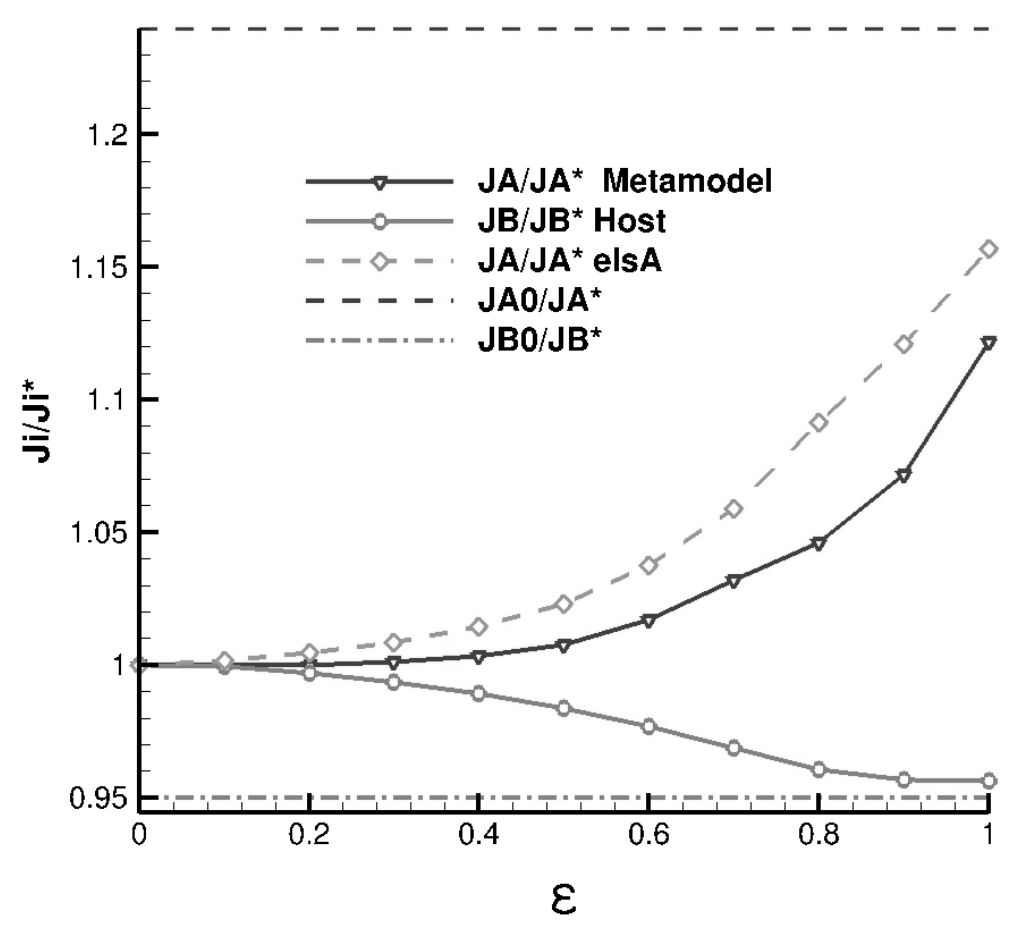

Fig. 10 Nash Game involving twist distribution.

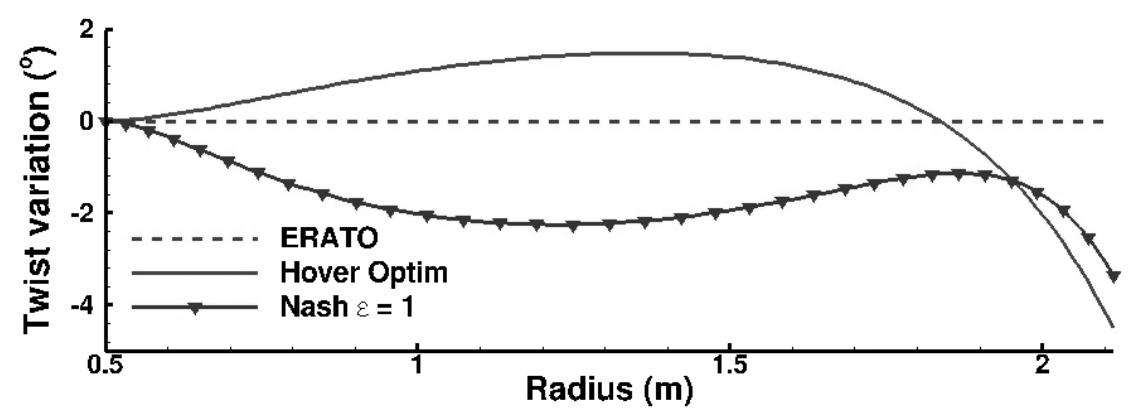

Fig. 11 Geometric twist variations of the hover optimum and the Nash equilibrium at $\epsilon=1$ with respect to the ERATO reference rotor. 
the rotor tip zone for practically all the azimuths (excepting the $\psi=90^{\circ}$ area, where the induced power is more important) as well as at the inner part of the blade for $\psi=330^{\circ}$. However, increased power is needed for the $0.6-0.8 \mathrm{R}$ zone at the fore blade. In addition, strong variations of lift and drag are observed in the advancing blade (where negative lift is produced at the blade tip). The rotor corresponding to the Nash equilibrium at $\epsilon=1$ represents a compromise between the baseline and the hover optimum. The power repartition is much more similar to the ERATO one, with a slight increase near the blade tip in the retreating side, while reducing the necessary power mainly in the inner part of the disk. In addition, the lift coefficient is more evenly distributed, with overall gains on the advancing side and almost the same drag repartition, which accounts for the Figure of Merit increase. The main differences are due to the slight reduction of twist at the blade tip while maintaining a similar distribution elsewhere.

\section{Sweep Optimization}

Constrained and Nash Games were conducted for the sweep distribution. The sweep laws are defined via a cubic spline with 5 control points. As presented in the previous section, a surrogate model was used to compute the Hessian and to substitute the CFD evaluations. The metamodel used 40 LHS samples plus 11 update points including the hover optimum. Again, 2 variables were assigned for the hover optimization and 3 for the forward flight.

The results on the unconstrained Nash Game are shown in Fig. 13. A very important reduction in power consumption is observed (2.2\% improvement with respect the ERATO rotor, and $6.7 \%$ with respect to the hover optimum), at a moderate penalty for the hover performance up to $\epsilon=0.9$. In reality, unconstrained optimizations tend to result in blades with a very high forward sweep angles or very unrealistic s-shaped blades with extreme variations. This kind of configuration is known to be unstable regarding flight mechanics as well as being unfavorable to the pitch link loads in the case of strong forward sweep.

In consequence, a Nash Game with a constraint on the pitch link load admissible values is presented in Fig.14, using a split of territories that includes the constraint gradient at the optimum in order to obtain feasible descent directions. Much more modest gains on the forward flight performance are obtained in 


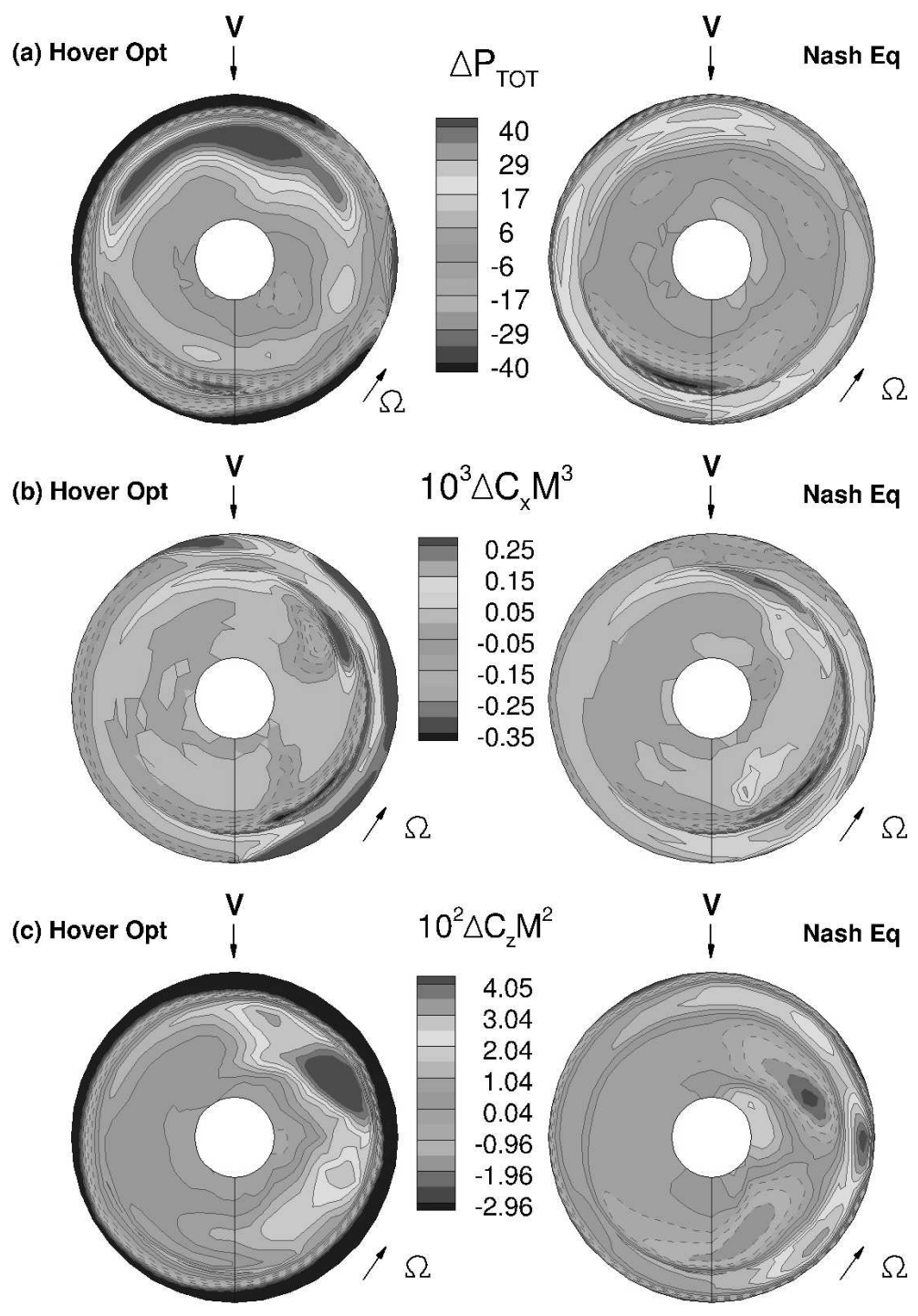

Fig. 12 Total power (a), sectional torque (b) and sectional lift (c) variations in forward flight of the hover optimum and the Nash compromise at $\epsilon=1$ with respect to ERATO (game including twist). 


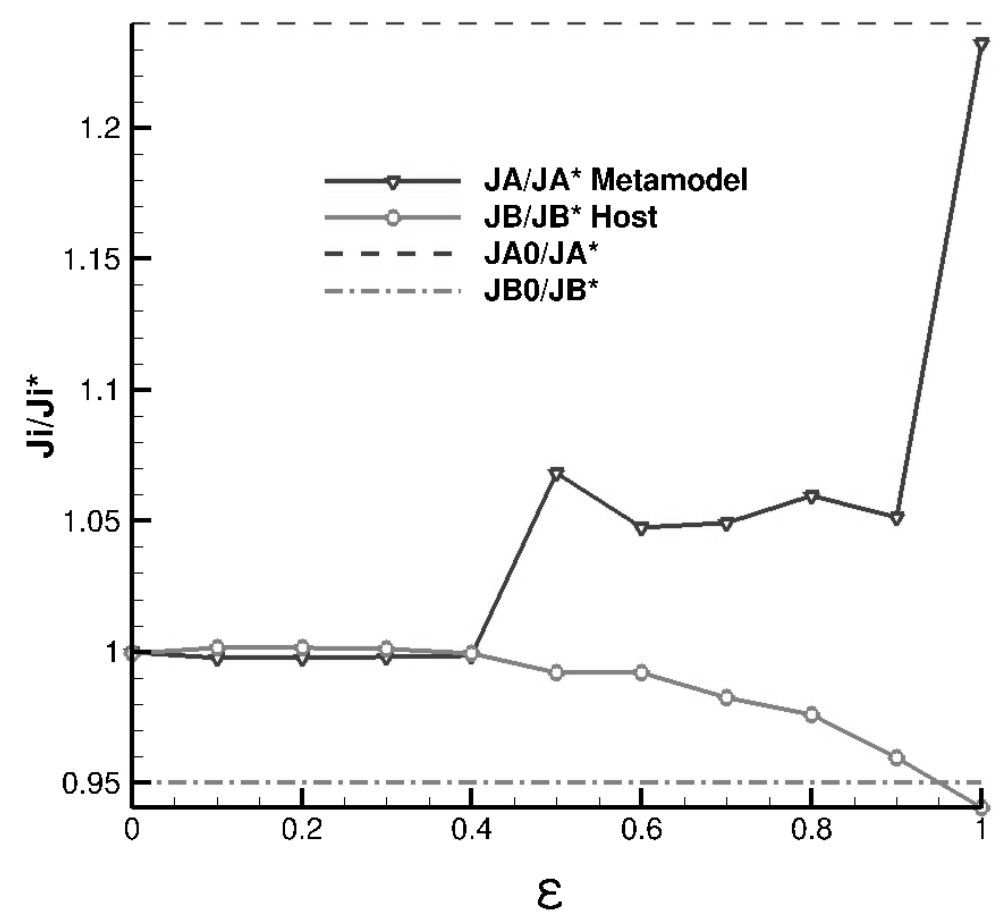

Fig. 13 Unconstrained sweep Nash Game evolution.

this case, due to the activation of the pitch link constraint. The optimized sweep law variation is presented in Fig. 15, still showing an exotic s-shaped blade. In this case, the pitch link loads are low due to the compensation of moments of the forward and backward swept zones, as in the blade presented by Brooks (Ref. 31). Additional shape constraints must be imposed in order to avoid this kind of configuration. This result highlights the importance of using higher fidelity models to evaluate the performance, specially in blades involving strong sweep which undergo important deformations. Weak coupling strategies along with the use of updated estimated structural properties and flight mechanics constraints seem necessary to obtain more realistic designs.

The forward flight performance variations of the Nash compromise with respect to ERATO are presented in Fig. 16 at $\epsilon=1$, computed using HOST. At this point, the power consumption is reduced by $2.9 \%$ with respect to the hover optimum (still consuming $+1.7 \%$ with regards to the original rotor) while conserving a gain of 5.9 points in the maximum value of the Figure of Merit. In this case the consumed power of the compromise solution is slightly reduced with respect to the hover optimum mainly in the fore 


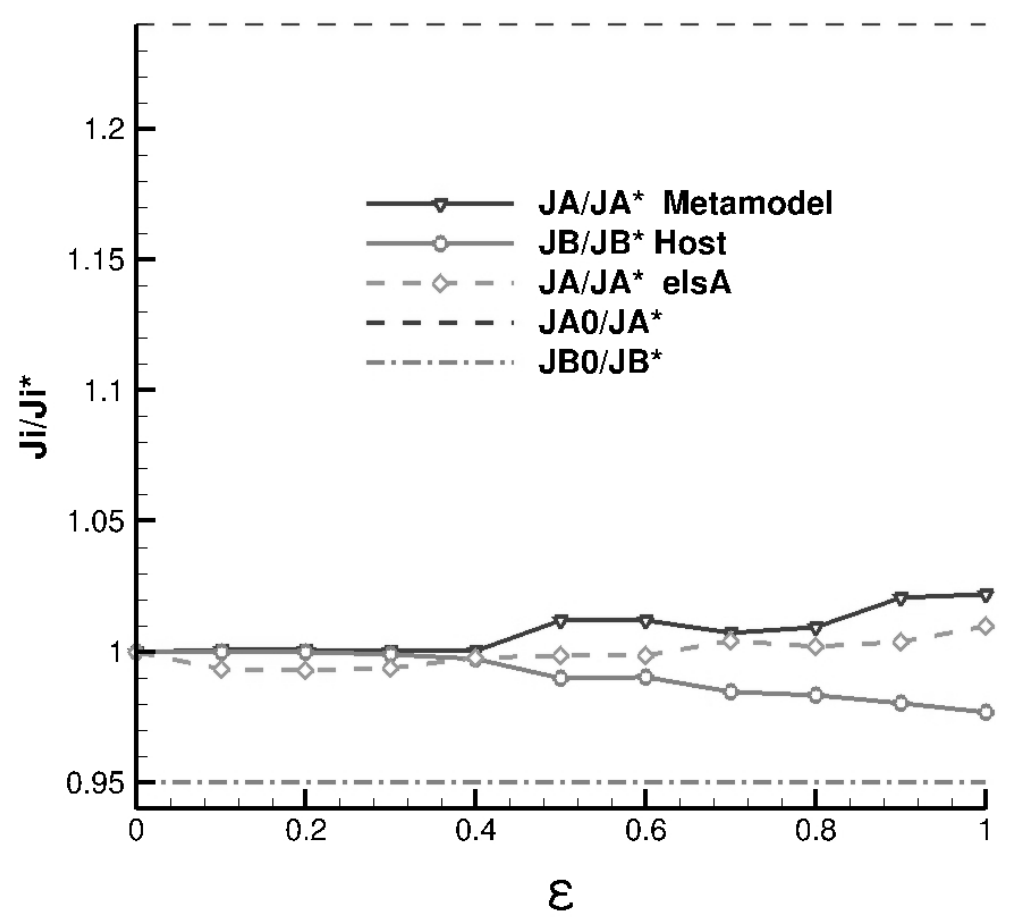

Fig. 14 Constrained sweep Nash Game evolution.

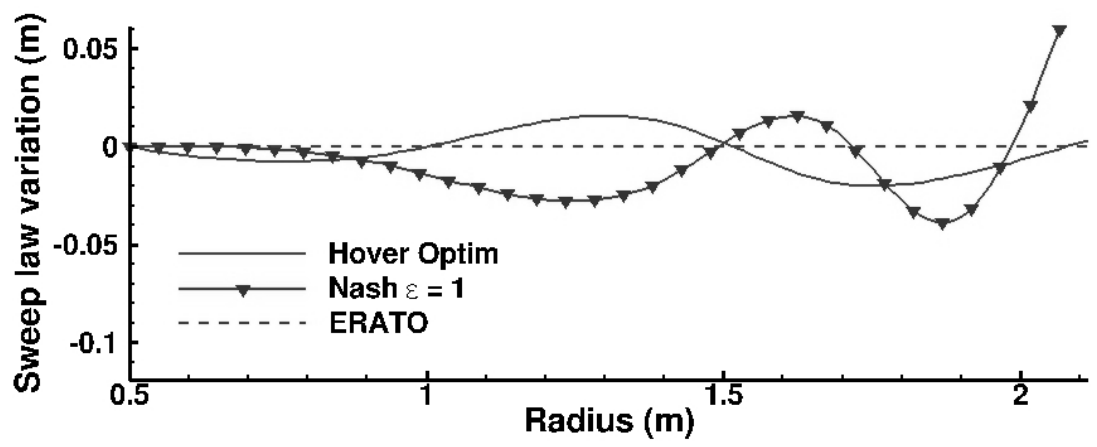

Fig. 15 Sweep law variations resulting from the constrained optimizations. 
blade zone $\psi=180^{\circ}$. The forward swept part at the blade tip seems to reduce the drag in the advancing part of the disk, while the lift is increased at the rotor tip (with respect to the hover optimum) and is overall more evenly distributed.

\section{Twist, Sweep and Chord Optimization}

A constrained Nash Game including twist, chord, sweep and collective pitch is presented in this section. The chord and twist laws were obtained using 5 Bézier poles, and the sweep was computed using a 5 point cubic spline. The collective is an additional variable only used in hover, as the rotor must be trimmed in forward flight. In total, 16 variables were used. The metamodel used to compute the hover performance and the Hessian at the optimum was built using a 150 samples LHS plus 22 additional points. In this case, 8 variables were assigned to each player. In addition, the constraint on the blade link loads was activated. The results of the Nash Game are shown in Fig. 17. The metamodel follows the similar trends as the CFD solver up to $\epsilon=0.7$, with an increasing error as the limits of the metamodel are reached. An important error in the computation of the equilibrium by the metamodel is obtained at $\epsilon=0.8$ and remains considerable up to $\epsilon=1$. In this case, the designer may consider that the equilibrium at $\epsilon=0.7$ offers a better compromise solution. The last equilibrium obtains a $1 \%$ power reduction with respect to the baseline ( $-5.5 \%$ w.r.t. the hover optimum) while conserving a gain of 2 points in the Figure of Merit.

In this case the chord is reduced at the blade tip zone with respect to the ERATO blade. The sweep distribution is also smoothed, straightening the blade, while the twist distribution follows the same trends as the baseline rotor with a slightly increased twist at the blade tip. The forward flight performance of the Nash equilibrium at $\epsilon=1$ is presented in Fig. 18. The power increase at the fore blade zone showed by the hover optimum is balanced for the Nash equilibrium, which reproduces the power distribution of the ERATO blade with a slight increase at $\psi=360^{\circ}$. As in the case presented for the twist Nash game, the twist reduction at the tip combined with the chord reduction in the same zone leads to an overall gain in lift in the advancing side. 


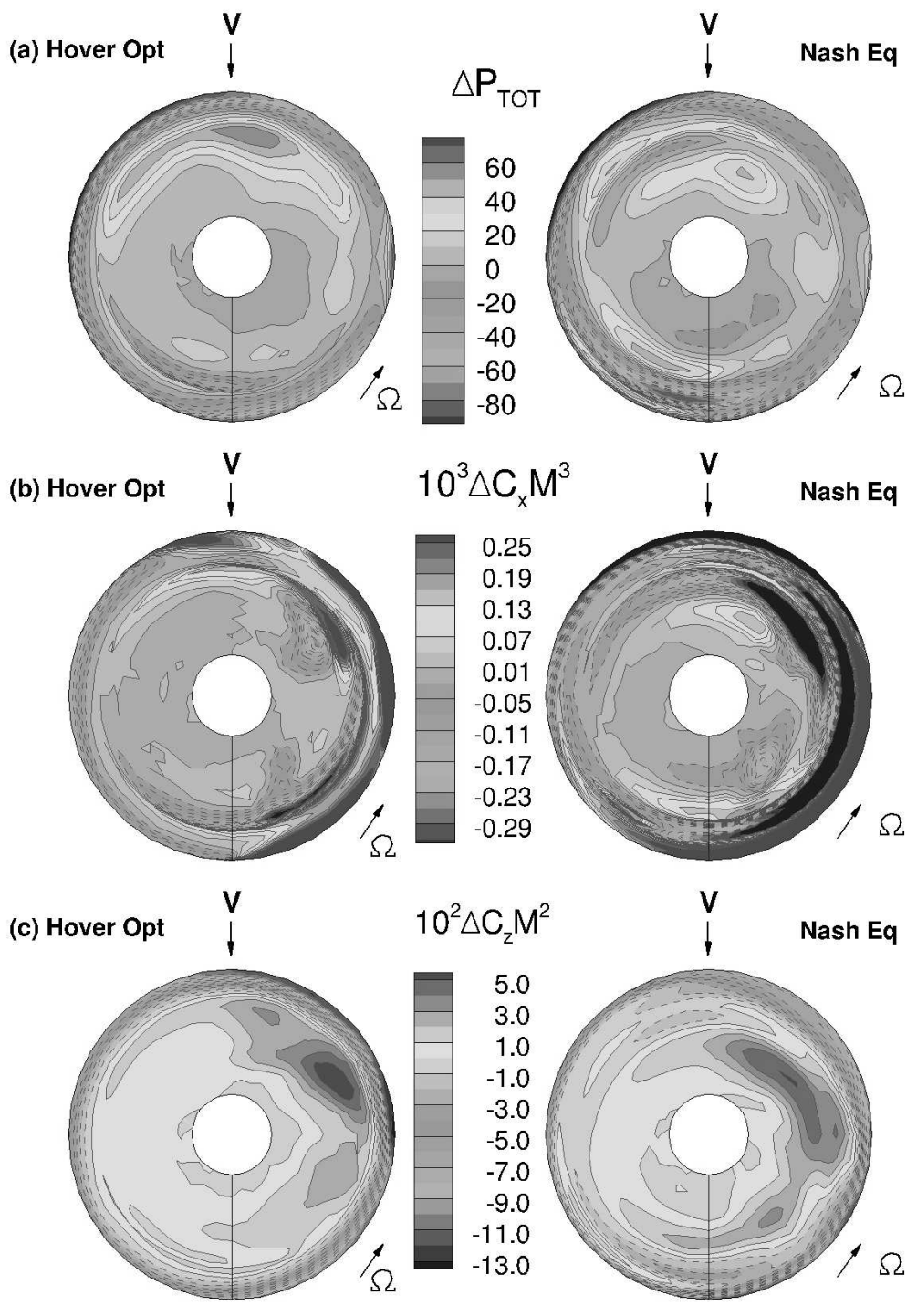

Fig. 16 Total power (a), sectional torque (b) and sectional lift (c) variations in forward flight of the hover optimum and the Nash compromise at $\epsilon=1$ with respect to ERATO (game including sweep). 


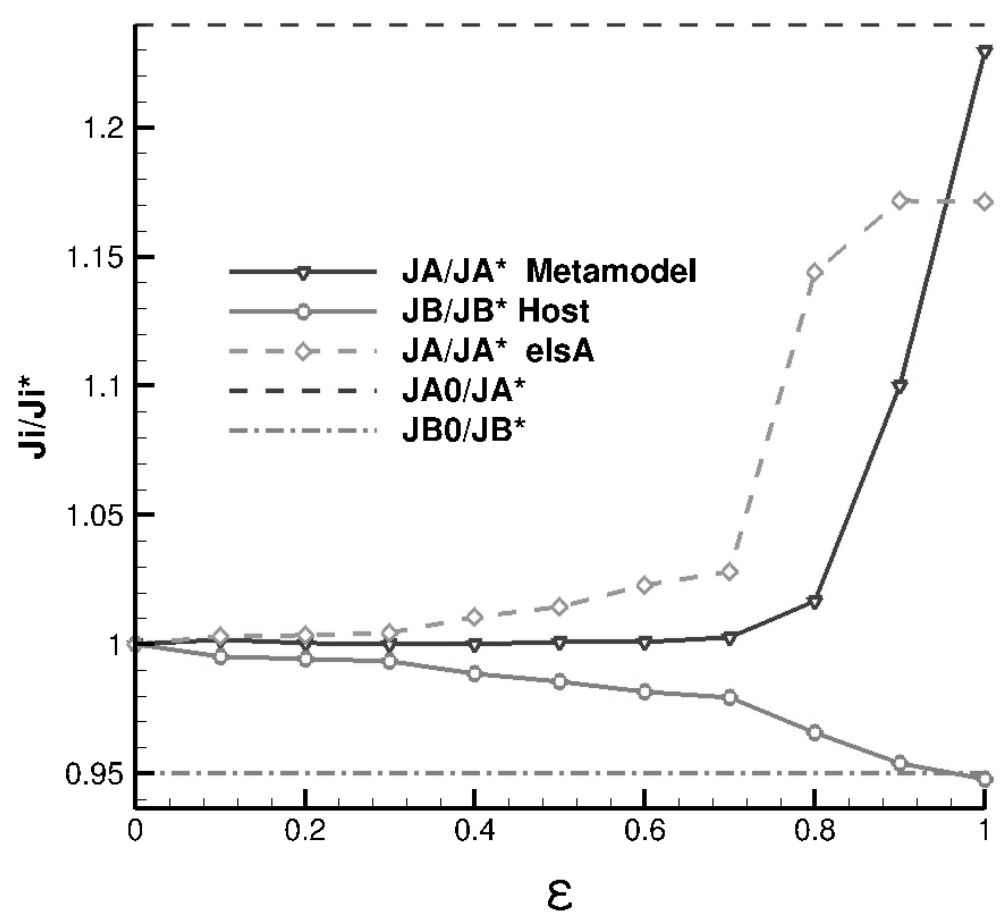

Fig. 17 Constrained sweep Nash Game evolution involving twist, chord and sweep. 


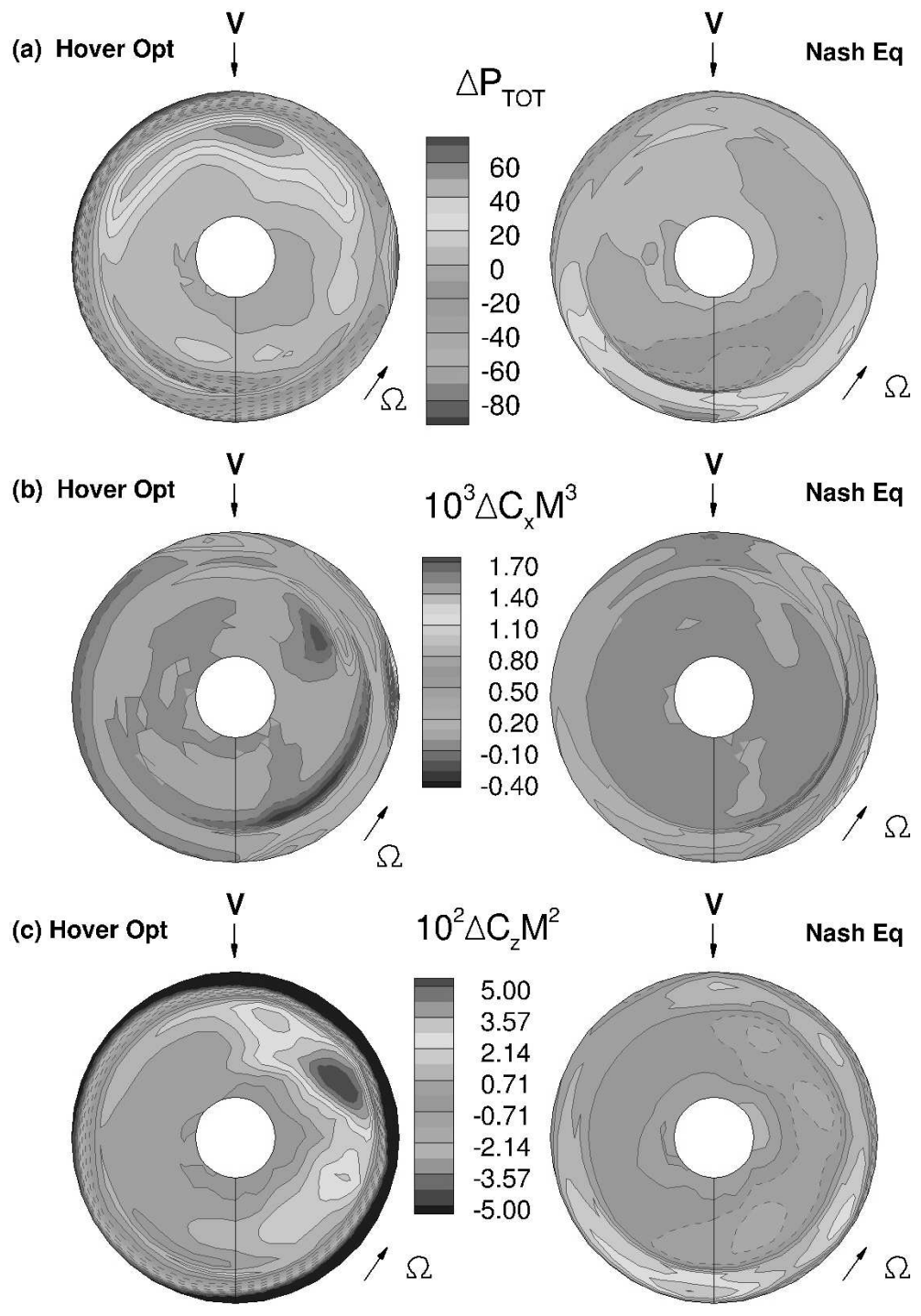

Fig. 18 Total power (a), sectional torque (b) and sectional lift (c) variations in forward flight of the hover optimum and the Nash compromise at $\epsilon=1$ with respect to ERATO (game including twist, chord and sweep). 


\section{Conclusions}

In this paper multi-objective shape optimizations of isolated rotors in hover and forward flight are presented. The objectives of the study were to maximize the FM and to minimize the required rotor power. A single objective optimization in hover was initially performed using a discrete adjoint solver and the CFD code elsA along with the gradient based algorithm CONMIN. The twist, chord and sweep distributions were parametrized using control points for Bézier curves and splines, used as variables. This method provided fast convergence to a local optimum using few high-fidelity evaluations.

An optimization strategy based on Nash Games is presented, combining the classical formulation with an innovative methodology to systematically split the design space to perform multi-objective concurrent optimizations. This method is adapted for cases where the objective functions are strongly antagonistic and a primary objective must not be excessively degraded. This strategy was then validated on the ERATO model rotor using twist, chord and sweep distribution optimizations starting from the previously optimized hover rotor. In order to reduce the computational costs of the objective evaluation in hover, metamodels were generated employing Design of Experiments and kriging techniques, while the forward flight performance was computed using the comprehensive rotor code HOST.

The obtained hover optimum showed a 6 points increment in the FM along with better capabilities at high rotor thrust. However, consumed power was significantly increased. Three Nash games are presented: the first one uses only the twist law to optimize the power, obtaining a compromise rotor with the same consumption as the baseline rotor and an increased FM employing 140 function evaluations and 20 gradient evaluations. The second Nash game involving sweep distributions obtains smaller gains in power consumption when activating pitch link load constraints, maintaining the gains obtained in the hover optimization. However, the optimization yields unrealistic s-shaped blades. This highlights the necessity to introduce higher fidelity models in forward flight evaluations to take into account 3D effects and blade deformations as well as structural and flight mechanics constraints to avoid unfeasible solutions. Finally, a constrained Nash game including chord, twist and sweep variations as variables was conducted. In this case the obtained rotors at the last 2 Nash equilibria optimized slightly both objectives at the same time. These results provide a methodology to conduct concurrent optimizations that provides a systematic 
hierarchy of solutions based on a physical split of the design space.

\section{References}

${ }^{1}$ Walsh, J., G.J., B., and Riley, M., "Optimization Methods Applied to the Aerodynamic Design of Helicopter Rotor Blades," Paper AIAA 2000-2013, 26st AIAA/ASME/ASCE/AHS Structures, Structural Dynamics and Materials Conference, Orlando, April 1985.

${ }^{2}$ Zibi, J., Defresne, G., and Costes, M., "A numerical procedure for aerodynamic optimization of helicopter rotor blades," 18th European Rotorcraft Forum Proceedings, Avignon, France, September 15$18,1992$.

${ }^{3}$ Le Pape, A. and Beaumier, P., "Numerical optimization of helicopter rotor aerodynamic performance in hover," Aerospace Science and Technology, Vol. 9, (3), 2005, pp. 191 - 201.

doi: 10.1016/j.ast.2004.09.004

${ }^{4}$ Dumont, A., Le Pape, A., Peter, J., and Huberson, S., “Aerodynamic Shape Optimization of Hovering Rotors Using a Discrete Adjoint of the Reynolds-Averaged NavierStokes Equations," Journal of the American Helicopter Society, Vol. 56, (3), 2011, pp. 1-11.

doi: doi:10.4050/JAHS.56.032002

${ }^{5}$ Choi, S., Potsdam, M., Lee, K., Iaccarino, G., and Alonso, J. J., "Helicopter rotor design using a time-spectral and adjoint-based method," AIAA Paper, 2008.

doi: $10.2514 / 6.2008-5810$

${ }^{6}$ Imiela, M., "High-fidelity optimization framework for helicopter rotors," Aerospace Science and Technology, December 2011.

doi: 10.1016/j.ast.2011.12.011

7Johnson, C. and Barakos, G., “Optimising Aspects of Rotor Blades in Forward Flight,”, 2011. doi: $10.2514 / 6.2011$

${ }^{8}$ Glaz, B., Friedmann, P. P., and Liu, L., "Surrogate based optimization of helicopter rotor blades for vibration reduction in forward flight," Structural and Multidisciplinary Optimization, Vol. 35, (4), June 2007, pp. 341-363. 
${ }^{9}$ Glaz, B., Friedmann, P. P., and Liu, L., "Helicopter Vibration Reduction throughout the Entire Flight Envelope Using Surrogate-Based Optimization," Journal of the American Helicopter Society, Vol. 54, (1), 2009, pp. 12007-1-12007-15.

doi: doi:10.4050/JAHS.54.012007

${ }^{10}$ Leusink, D., Alfano, D., Cinnella, P., and Robinet, J.-C., "Aerodynamic rotor blade optimization at Eurocopter-a new way of industrial rotor blade design," 51st AIAA Aerospace Sciences Meeting including the New Horizons Forum and Aerospace Exposition, 2013.

doi: $10.2514 / 6.2013-779$

${ }^{11}$ Le Pape, A., "Numerical Aerodynamic Optimization of Helicopter Rotors: Multiobjective Optimization in Hover and Forward Flight Conditions," 31st European Rotorcraft Forum Proceedings, Florence, Italy, September 2005.

${ }^{12}$ Nash, J., "Non-cooperative games," Annals of mathematics, Vol. 54, (2), 1951, pp. 286-295.

${ }^{13}$ Prieur, J. and Splettstoesser, W. R., "ERATO - An ONERA-DLR Cooperative Programme On Aeroacoustic Rotor Optimization,” 25th European Rotorcraft Forum, Rome, Italy, September 1999.

${ }^{14}$ Cambier, L. and Veuillot, J., "Status of the elsA CFD software for flow simulation and multidisciplinary applications," 46th AIAA Aerospace Science Meeting and Exhibit, Vol. 664, 2008.

${ }^{15}$ Benoit, B., Dequin, A., Kampa, K., Von Grünhagen, W., Basset, P., and Gimonet, B., "Host, a General Helicopter Simulation Tool for Germany and France," American Helicopter Society 56th Annual Forum, Virginia Beach, USA, May 2000.

${ }^{16}$ Vanderplaats, G. N., "CONMIN: A FORTRAN program for constrained function minimization," Technical report, NASA, NASA-TM-X-62282, August 1973.

${ }^{17}$ Adams, B., Bohnhoff, W., Dalbey, K., Eddy, J., Eldred, M., Gay, D., Haskell, K., Hough, P., and Swiler, L., DAKOTA, A Multilevel Parallel Object-Oriented Framework for Design Optimization, Parameter Estimation, Uncertainty Quantification, and Sensitivity Analysis: Version 5.0 User's Manual, Sandia National Laboratories Albuquerque, NM, Sandia Technical Report SAND2010-2183, December 2009.

${ }^{18}$ Tang, Z., Désidéri, J.-A., and Périaux, J., "Multi-criterion Aerodynamic Shape-Design Optimization and Inverse Problems Using Control Theory and Nash Games," Journal of Optimization Theory and Applications, Vol. 135, (1), October 2007. 
${ }^{19}$ Désidéri, J.-A., "Cooperation and competition in multidisciplinary optimization,” Computational Optimization and Applications, Vol. 52, 2012, pp. 29-68.

doi: 10.1007/s10589-011-9395-1

${ }^{20} \mathrm{Kok}$, J. C., "Resolving the dependence on freestream values for the k-omega turbulence model," AIAA journal, Vol. 38, (7), 2000, pp. 1292-1295.

${ }^{21}$ Menter, F., "Two-equation eddy-viscosity turbulence models for engineering applications," AIAA Journal, Vol. 32, (8), 1994, pp. 1598-1605.

${ }^{22}$ Roe, P. L., “Approximate Riemann solvers, parameter vectors, and difference schemes,” Journal of computational physics, Vol. 43, (2), 1981, pp. 357-372.

${ }^{23}$ Van Albada, G., Van Leer, B., and Roberts Jr, W., “A comparative study of computational methods in cosmic gas dynamics," Astronomy and Astrophysics, Vol. 108, 1982, pp. 76-84.

${ }^{24}$ Arnaud, G. and Beaumier, P., "Validation of R 85/METAR on the Puma RAE flight tests," ONERA, 1992, pp. 11.

${ }^{25}$ McKay, M. D., Beckman, R. J., and Conover, W. J., “Comparison of three methods for selecting values of input variables in the analysis of output from a computer code," Technometrics, Vol. 21, (2), 1979, pp. 239-245.

${ }^{26}$ Mistree, F., Korte, J. J., Mauery, T. M., and Simpson, T. W., "Kriging models for global approximation in simulation-based multidisciplinary design optimization," AIAA journal, Vol. 39, (12), 2001.

${ }^{27}$ Kohavi, R., "A study of cross-validation and bootstrap for accuracy estimation and model selection," International joint Conference on artificial intelligence, Vol. 14, 1995.

${ }^{28}$ Diamantidis, N., Karlis, D., and Giakoumakis, E., "Unsupervised stratification of cross-validation for accuracy estimation,” Artificial Intelligence, Vol. 116, (1), 2000, pp. 1-16.

${ }^{29}$ Jones, D. R., Schonlau, M., and Welch, W. J., "Efficient global optimization of expensive black-box functions," Journal of Global optimization, Vol. 13, (4), 1998, pp. 455-492.

${ }^{30}$ Bingham, G. J., "The Aerodynamic Influences of Rotor Blade Taper, Twist, Airfoils and Solidity on Hover and Forward Flight Performance.” Technical report, DTIC Document, 1982.

${ }^{31}$ Brooks, T. F., “Forward sweep, low noise rotor blade," US Patent 5.584.661, December 1996. 


\section{List of Figures}

1 Flow chart of the optimization process. . . . . . . . . . . . . . . . . . . 10

2 Mesh strategy and ERATO blade geometry. . . . . . . . . . . . . . . . . . . . . 13

3 Control point locations for the ERATO blade optimization. . . . . . . . . . . . . . . 14

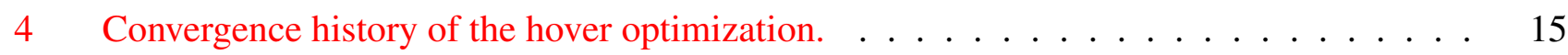

5 Geometric twist, chord and sweep law variations of the optimum (continuous line) with respect to the baseline rotor (dashed line) . . . . . . . . . . . . . . 16

6 Maximum FM gain for the optimum with respect to the baseline rotor. . . . . . . . . .

7 Lift and power distribution along the blade span of the baseline and optimized rotor at the same thrust value. . . . . . . . . . . . . . . . . . . . . . . . 18

8 Pressure coefficient contours for the baseline and optimized rotor at the same thrust value. 18

9 Friction lines comparison at the blade tip for the thrust corresponding to the baseline $F M_{M A X}$ and at the optimized blade $F M_{M A X} \ldots \ldots \ldots \ldots$

10 Nash Game involving twist distribution. . . . . . . . . . . . . . . . . . . . 21

11 Geometric twist variations of the hover optimum and the Nash equilibrium at $\epsilon=1$ with respect to the ERATO reference rotor. $\ldots \ldots \ldots \ldots \ldots \ldots$

12 Total power (a), sectional torque (b) and sectional lift (c) variations in forward flight of the hover optimum and the Nash compromise at $\epsilon=1$ with respect to ERATO (game

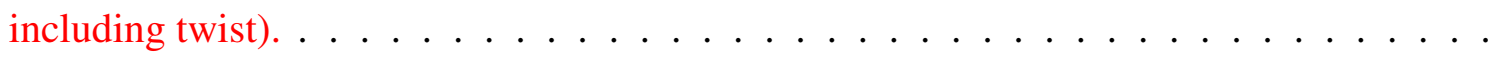

13 Unconstrained sweep Nash Game evolution. . . . . . . . . . . . . . . . . . . . . . . 24

14 Constrained sweep Nash Game evolution. . . . . . . . . . . . . . . . . . . . . 25

15 Sweep law variations resulting from the constrained optimizations. . . . . . . . . . . . 25

16 Total power (a), sectional torque (b) and sectional lift (c) variations in forward flight of the hover optimum and the Nash compromise at $\epsilon=1$ with respect to ERATO (game including sweep). . . . . . . . . . . . . . . . . . . . . . . 27

17 Constrained sweep Nash Game evolution involving twist, chord and sweep. . . . . . . . 28 
18 Total power (a), sectional torque (b) and sectional lift (c) variations in forward flight of the hover optimum and the Nash compromise at $\epsilon=1$ with respect to ERATO (game including twist, chord and sweep). . . . . . . . . . . . . . . . 